\title{
Cystic fibrosis and survival to 40 years: a study of cystic fibrosis transmembrane conductance regulator function
}

\author{
N.J. Simmonds*, L. D'Souza*, M. Roughton`, E.W.F.W. Alton+, \\ J.C. Davies ${ }^{+, \S}$ and M.E. Hodson*
}

ABSTRACT: Significant survival heterogeneity exists in cystic fibrosis. Our aim was to determine whether residual function of the cystic fibrosis transmembrane conductance regulator (CFTR) is present in long-term survivors with severe mutations.

Nasal potential difference (PD) and sweat chloride were measured in $\mathbf{3 4}$ long-term survivors (aged $\geqslant 40 \mathrm{yrs}$ ) and compared with young patients (18-23 yrs) with severe $(n=30)$ and mild $(n=31)$ lung disease.

Baseline PD was not significantly different across the three groups (long-term survivors, -42.8 (range -71.0- -20.5) mV; young/mild, -40.5 (-58.8- -19.5) mV; young/severe,-46.3 (-74.0- -20.0) mV). Response to amiloride ( $\Delta \mathrm{Amil})$ was significantly different across the three groups $(p=0.01)$; longterm survivors had values (27.8 (range 8.5-46) $\mathrm{mV}$ ) which were not different to either young group, but the young/severe group had significantly higher values $(29.5(11-47) \mathrm{mV})$ than those in the young/mild group (22.0 (7-39) $\mathrm{mV} ; \mathbf{p}<0.01)$. Baseline PD and $\triangle$ Amil were associated with forced expiratory volume in $1 \mathrm{~s}$ (FEV1) (co-efficient $(95 \% \mathrm{Cl})-0.13(-0.23--0.03) ; p=0.009$ and -0.12 $(-0.20-0.04) ; p=0.003$, respectively). Sweat chloride was lowest $(p<0.05)$ in the young/severe group (93.5 (74-111) $\mathrm{mmol} \cdot \mathrm{L}^{-1}$ versus $98.8(76.5-116.0) \mathrm{mmol} \cdot \mathrm{L}^{-1}$; long-term survivors; and 99.5 (80.0-113.5) $\mathrm{mmol} \cdot \mathrm{L}^{-1}$; young/mild).

$\triangle \mathrm{Amil}$ is associated with $\mathrm{FEV}_{1}$ but our findings indicate that long-term survival cannot be explained by residual CFTR function when measurements are taken in later life.

KEYWORDS: Cystic fibrosis, forced expiratory volume in $1 \mathrm{~s}$, nasal potential difference, sweat chloride, survival

ystic fibrosis (CF) is an autosomal recessive condition caused by mutations of the cystic fibrosis transmembrane conductance regulator (CFTR) gene and characterised by abnormal airway ion transport and raised sweat chloride [1]. The gene is responsible for the production of CFTR protein which functions as a cyclic adenosine monophosphate (cAMP)-dependent chloride channel in epithelial cells, as well as regulating sodium transport in the airways via the epithelial sodium channel $(\mathrm{ENaC})[2,3]$. Disruption of this protein in $\mathrm{CF}$ results in diminished chloride secretion and sodium hyperabsorption, abnormalities which can be assessed by nasal potential difference (PD) measurements $[4,5]$.

The genetic mutations responsible for CF are classified by their functional capacity with class I to III mutations generally considered as "severe" defects. However, specific genotypes correlate poorly with pulmonary function, and disease heterogeneity exists for individuals with severe mutations [6, 7]. Putative mechanisms for this include genetic modifiers and environmental factors [8]. Sporadic residual chloride function has also been demonstrated in homozygous $\Delta F 508$ (a class II mutation) patients by nasal PD measurement [9-11].

As the pathological manifestations of CF probably result from abnormal epithelial ion transport, it follows that variations in disease expression (and ultimately survival) may be due to variations of CFTR function. We have recently reported $112 \mathrm{CF}$ patients $\geqslant 40$ yrs of age, of whom $30 \%$ were homozygous $\Delta \mathrm{F} 508$ and many were relatively clinically stable [12], and in this population only limited evidence for environmental influences exist, suggesting that CFTR function variability might be important [13]. Some studies have demonstrated that lower baseline nasal PD [4, 14], a decreased response to amiloride [15] and lower sweat chloride
AFFILIATIONS

*Dept of Cystic Fibrosis, Royal Brompton Hospital and Imperial College,

\#Dept of Biochemistry, Royal Brompton Hospital,

"Dept of Health Services Research,

Royal Brompton Hospital,

${ }^{\text {s}}$ Dept of Paediatric Respiratory Medicine, Royal Brompton Hospital, and

+Dept of Gene Therapy, National Heart and Lung Institute, Imperial College, London, UK.

CORRESPONDENCE

N.J. Simmonds

Dept of Cystic Fibrosis

Royal Brompton Hospital

Sydney Street

London

SW3 6NP

UK

E-mail: n.simmonds@imperial.ac.uk

Received:

May 202010

Accepted after revision:

Aug 182010

First published online:

Sept 162010 
concentrations [16] are associated with mutations linked to milder phenotypes (e.g. pancreatic sufficiency) and others have shown that ion transport abnormalities have distinct clinical outcomes [11]. No study has yet determined if preserved CFTR function confers a significant survival advantage in patients with "severe" mutations.

In view of the evidence relating CFTR function variability with clinical status and the importance of identifying mechanisms underlying survival advantages, we prospectively evaluated the transepithelial nasal PD and sweat chloride of long-term survivors of CF with severe mutations, hypothesising that their survival advantage was, in part, due to residual CFTR function. Young CF adults with severe and mild lung disease were used as controls and compared with CF adults $\geqslant 40$ yrs of age; we postulated that the long-term survivors would have CFTR function similar to the young mild group, and both would be significantly better than the young severe group.

\section{METHODS}

\section{Subjects}

The inclusion criteria for this study were: 1) a definitive diagnosis of CF (characteristic clinical manifestations and confirmatory diagnostic tests) [17]; 2) a disease-causing CFTR mutation, class I to III only and on both alleles; 3) aged $18-23$ yrs (controls) or $\geqslant 40$ yrs (long-term survivors). Lung function (forced expiratory volume in $1 \mathrm{~s}$ (FEV1) and forced vital capacity (FVC)) was collected retrospectively for all patients so that the younger group could be subdivided into terciles by FEV1. It was taken from their most recent annual review assessment (i.e. within 12 months). This had been performed during clinical stability, using a standard spirometer (Jaeger, Würzburg, Germany) and expressed as \% predicted for age, sex and height using published reference values [18]. Of the young patient group, only those in the lower and upper FEV1 terciles were recruited. There were no lung function restriction criteria for the older group. Patients were excluded from PD measurements if they had nasal polyposis $(n=1)$ or recent nasal surgery $(n=1)$. The measurement was deferred if they had an acute upper respiratory tract infection/ rhinitis (by 1 month after full symptom resolution). Informed, written consent was obtained from all participants. The study was approved by the Royal Brompton Hospital Research Ethics Committee (London, UK).

\section{Nasal PD and sweat chloride}

Nasal PD measurement was performed by a single operator using the method described by MidDLETON et al. [19]. The integrity of the silver/silver chloride electrodes was verified by measuring finger PD. PD measurements were performed with perfusion of solutions at room temperature in the following sequence: 1) maximal (most negative) stable baseline PD in the presence of a buffer solution (HEPES); 2) perfusion with amiloride (change in PD in the presence of amiloride $(\triangle \mathrm{Amil})$ ); 3) perfusion with a low chloride solution (change in PD in the presence of a low chloride solution $\left(\Delta\right.$ low $\left.\mathrm{Cl}^{-}\right)$); and 4) perfusion with isoprenaline (Iso) in a low chloride solution. These stages represent sodium channel-mediated sodium hyperabsorption (1 and 2), hyperpolarisation via chloride secretion occurring down an electrochemical gradient (3) and activation of cAMP-dependent chloride (CFTR) channels (4).
Steps 3 and 4 together measure the change in PD in the presence of low chloride solution followed by low chloride/Iso solution ( $\Delta$ low $\mathrm{Cl}^{-}+$Iso). To take into account both the sodium and chloride elements of the nasal PD measurements, the Wilschanski index was calculated (index $=\mathrm{e}^{\text {response to low }}$ chloride solution and isoprenaline/response amiloride) [20]; a cut-off $>0.70$ predicts CF. The nostril with the highest (most negative) baseline was studied. For internal consistency, PD measurement was repeated in the same nostril at the point of highest baseline and at least $6 \mathrm{~h}$ apart (duration chosen to allow the dissipation of residual amiloride and Iso based on prior experiments (unpublished data)). Two sweat tests were performed using the Macroduct system [21] and analysed using a titrimetric assay with silver nitrate reagent. They were both performed on the same day (which was the same day as the first nasal PD measurement) under identical conditions.

\section{Clinical data}

Body mass index (BMI) was taken from the patients' most recent annual review record. BMI and spirometry were also taken from the first available records of the old group (i.e. when they would have been a similar age to the young groups). The remaining clinical information was taken from the CF database of the Royal Brompton Hospital (London, UK): pancreatic insufficiency (low faecal elastase; $<200 \mu \mathrm{g} \cdot \mathrm{g}^{-1}$ ), CF-related diabetes (CFRD) (based on oral glucose tolerance test and serial glucose monitoring); and sputum microbiology (chronic infection, $75 \%$ of sputum samples positive for the organism over the previous 12 months based on more than four samples).

\section{Statistical analysis}

A sample size of 34 patients in each group provides $80 \%$ power testing at the $5 \%$ significance level using one-way ANOVA to compare the baseline nasal PD between the three groups (based on expected baseline \pm SD values of $-35 \pm 9 \mathrm{mV}$ (young/ severe) and $-29 \pm 9 \mathrm{mV}$ (young/mild and old)). Patient permitting, nasal PD and sweat chloride measurements were repeated to reduce intra-subject variability. If repeat measurements were performed, the arithmetic mean for each patient was calculated. Differences in nasal PD and sweat test measurements were tested by Kruskal-Wallis (for non-parametric data) and then if significant, by Dunn's multiple comparison. Fisher's exact test was used to compare categorical variables. Linear regression models were used to assess the relationship between continuous variables. Multivariate analyses were performed with FEV1, BMI, Pseudomonas aeruginosa status and age as independent variables. A paired t-test was used to assess the change in BMI and spirometry in the old group from the first to most recent assessment. A p-value of $<0.05$ was considered to be statistically significant.

\section{RESULTS}

\section{Clinical characteristics}

Nasal PD measurements and sweat chloride concentrations were prospectively measured for 94 patients with CF. The clinical characteristics of each group are presented in table 1. Older patient data are presented as the most recent and the first recorded value (at mean $\pm \mathrm{SD}$ age $22.8 \pm 8.1 \mathrm{yrs}$, i.e. similar to the young groups, mean \pm SD age $21.1 \pm 1.8 \mathrm{yrs}) .76(80.9 \%)$ patients were homozygous $\Delta \mathrm{F} 508$ and the remainder were $\Delta$ F508 compound heterozygotes (with genotype G551D $(n=5)$, 


\begin{tabular}{|c|c|c|c|c|}
\hline Clinical details & Old & Young/severe & Young/mild & $p$-value \\
\hline Subjects $n$ & 33 & 30 & 31 & \\
\hline Age yrs & $44(40.2-57.5)$ & $21.6(18.8-23.9)$ & $20.2(18-23.9)$ & \\
\hline Male & 21 (63.6) & $17(56.7)$ & $23(74)$ & 0.35 \\
\hline PI & $33(100)$ & $30(100)$ & $29(93.5)^{\#}$ & 0.13 \\
\hline CFRD & $15(45.5)$ & 7 (23.3) & $3(9.7)$ & 0.005 \\
\hline \multicolumn{5}{|l|}{ Microbiology } \\
\hline S. aureus & $16(48.5)$ & 7 (23.3) & $12(38.7)$ & 0.12 \\
\hline$P$. aeruginosa & $28(84.8)$ & 28 (93.3) & $17(54.8)$ & 0.001 \\
\hline B. cepacia & $2(6.1)$ & 0 & $3(9.7)$ & 0.24 \\
\hline S. maltophilia & 0 & $2(6.7)$ & $1(3.2)$ & 0.32 \\
\hline MRSA & $1(3.0)$ & $2(6.7)$ & $1(3.2)$ & 0.74 \\
\hline \multirow[t]{2}{*}{ FEV $1 \%$ pred } & $49.5 \pm 20.9$ & $40.9 \pm 10.9$ & $83.6 \pm 13.9$ & $<0.05$ \\
\hline & $66.1 \pm 27.0$ & & & $<0.05$ \\
\hline \multirow[t]{2}{*}{ FVC $\%$ pred } & $77.5 \pm 20.6$ & $61.8 \pm 11.1$ & $97.5 \pm 11.7$ & $<0.05$ \\
\hline & $77.8 \pm 22.8$ & & & \\
\hline \multirow[t]{2}{*}{$\mathrm{BMI} \mathbf{k g} \cdot \mathrm{m}^{-2}$} & $22.8 \pm 4.0$ & $19.0 \pm 2.1$ & $21.8 \pm 3.0$ & $<0.05$ \\
\hline & $19.6 \pm 1.8$ & & & \\
\hline
\end{tabular}

Data are presented as median (range), $\mathrm{n}(\%)$ or mean $\pm \mathrm{SD}$, unless otherwise stated. PI: pancreatic insufficient; CFRD: cystic fibrosis-related diabetes; S. aureus: Staphylococcus aureus; P. aeruginosa: Pseudomonas aeruginosa; B. cepacia: Burkholderia cepacia; S. maltophilia: Stenotrophomonas maltophilia; MRSA: methicillin-resistant $S$. aureus; FEV1: forced expiratory volume in $1 \mathrm{~s}$; $\%$ pred: \% predicted; FVC: forced vital capacity; BMI: body mass index. \#: genotypes confirmed as $\Delta F 508 / \Delta F 508$ and $F 508 / G 551 D$. Values given in italics for FEV1, FVC and BMl are for the old group at initial presentation (mean age $22.8 \pm 8.1 \mathrm{yrs}$ ) to the adult cystic fibrosis clinic.

G542X $(n=3), N 1303 K(n=3), 1717-1 G \rightarrow A(n=2), 621-1 G \rightarrow T$ $(n=1), \operatorname{R} 1162 X(n=1), 2789+3 \operatorname{delG}(n=1), 3659 \operatorname{delC}(n=1)$ and D1507 $(n=1)$ ); no significant difference between the groups for any genotype was present.

BMI was lowest in the young/severe group (table 1). In the older group, there was a significant $(\mathrm{p}<0.001)$ increase in BMI and decline $(p<0.001)$ in FEV1 over the period from the first recorded to the most recent value $($ mean \pm SD) $23.0 \pm 7.9$ yrs (table 1$)$. First recorded FEV1 was significantly $(p<0.001)$ greater than the young/severe group but lower $(\mathrm{p}<0.05)$ than young/mild group. First recorded BMI was similar to the young/severe group and significantly $(\mathrm{p}<0.01)$ lower than the young/mild group.

Neither sex ratio $(65 \%$ male) nor pancreatic status was different across the groups. Significant differences were seen with respect to frequency of CFRD (more common in the old versus young/mild group, $\mathrm{p}=0.005$ ) and chronic infection with $P$. aeruginosa (more common in the old and young/severe versus young/mild group; $\mathrm{p}<0.05$ and $\mathrm{p}<0.005$, respectively).

\section{Nasal PD measurements}

A total of 153 nasal PD measurements were performed $(93+60$ repeat measurements $(n=23$, old; $n=19$, young severe; and $\mathrm{n}=18$, young/mild)). Ion transport measurements for each group are shown in figure 1. Baseline PD was not significantly different across the three groups. $\Delta$ Amil was significantly different across the three groups ( $\mathrm{p}=0.01$, Kruskal-Wallis); the older group had values which were not significantly different to either young group but the young/severe group had higher values than those in the young/mild group $(\mathrm{p}<0.01)$. The responses to $\Delta \mathrm{lowCl}^{-}+$Iso were minimal; hyperpolarisation (i.e. towards normal (more negative)) did not exceed a mean of $1.2 \mathrm{mV}$ in any group (with no significant difference between the groups). $\Delta$ Amil values remained significantly different $(p<0.05)$ between the young/mild and young/severe group, and the $\Delta$ low $\mathrm{Cl}^{-}+$Iso response was unchanged when analysed separately for both sexes (data not shown).

On univariate analysis, baseline PD and $\triangle$ Amil (of all three groups) were both significantly associated with FEV1 (fig. 2), with coefficients $(95 \% \mathrm{CI})-0.13(-0.23--0.03) ; \mathrm{p}=0.009$ and $-0.12(-0.20--0.04) ; \mathrm{p}=0.003$, respectively. $\Delta \mathrm{lowCl}^{-}+$Iso was not associated with lung function and no PD measurement was associated with age or BMI on univariate analysis. When multivariate analysis was performed, FEV1, but not age, BMI or $P$. aeruginosa status were related to ion transport measurements (table 2).

\section{Patients with significant chloride secretion}

Seven patients demonstrated hyperpolarisation of at least $5 \mathrm{mV}\left(\Delta \mathrm{lowCl}^{-}+\right.$Iso) and, of those, three were over $10 \mathrm{mV}$, indicating chloride secretion well into the recognised "normal" range [22]. One of the three had a repeat measurement and $\Delta$ low $\mathrm{Cl}^{-}+$Iso remained significantly high $(-18$ and $-19 \mathrm{mV})$. The characteristics of the three patients are summarised in table 3 .

\section{Sweat tests}

Sweat chloride concentrations for each group are shown in figure 3. A total of 177 sweat tests were performed $(91+86$ repeat measurements). Sweat chloride concentrations were significantly different across the three groups $(\mathrm{p}<0.05$, Kruskal-Wallis); the young/mild group had values which were significantly higher $(p<0.05)$ than the young/severe group. On univariate analysis, sweat chloride concentration (of all three groups) was associated with FEV1 (coefficient $(95 \%$ CI) $0.74(0.20-1.29) ; \mathrm{p}=0.008)$, FVC (0.62 (0.15-1.10); $\mathrm{p}=0.011)$ and BMI $(0.16(0.08-0.24) ; \mathrm{p}<0.001)$, but not with age or nasal PD. On multivariate analysis, only BMI was associated with sweat chloride. The intra-subject variability with $95 \%$ limits of agreement for sweat chloride and nasal PD measurements is shown in table 4.

\section{DISCUSSION}

This prospective study of in vivo CFTR function in long-term survivors of CF with severe mutations demonstrates that neither nasal ion transport nor sweat chloride were associated with increased survival. However, there was an association between nasal PD and pulmonary disease severity, although this related only to sodium and not to chloride transport.

The explanations for these findings are likely to fall into two main categories. First, the study hypothesis relies on the fact that disease heterogeneity in patients with similar genotypes results primarily from factors influencing CFTR function. While it is well recognised that disease severity can vary greatly for identical mutations, it is also recognised that concordance is greater in monozygotic twins than dizygotic twins [23]. This indicates that "environmental" factors 

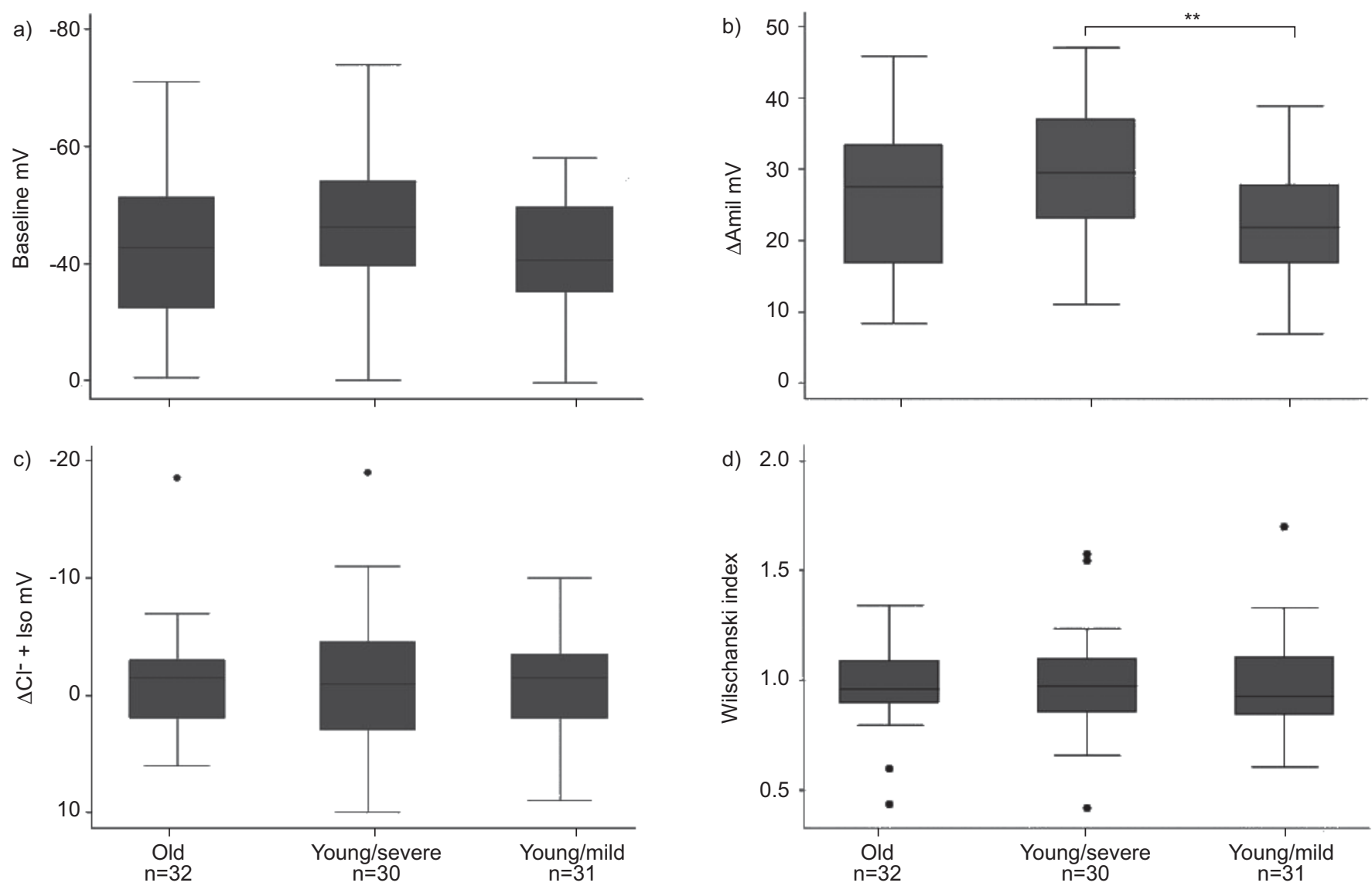

FIGURE 1. Box plots demonstrating the range and variability of nasal potential difference (PD) measurements. a) Baseline; $b$ ) change in PD in the presence of amiloride

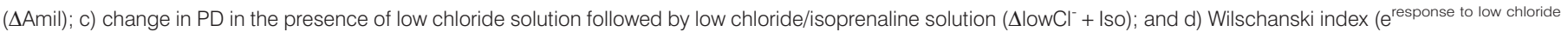
solution and isoprenaline/response amiloride) for each group. The values were significantly different across the three groups ( $p=0.01$, Kruskal-Wallis) for $\Delta$ Amil only; the older group had values which were not different to either young group but the young/severe group had higher values than those in the young/mild group. Boxes show the median and interquartile range (IQR). Whiskers show the highest and lowest data points within 1.5 IQR of the upper and lower quartile. $\bullet$ : outliers outside of these limits. ${ }^{\star}$ : $\mathrm{p}<0.01$.

(e.g. time of diagnosis and adherence to treatment) cannot be exclusively responsible for this variability and other genetic influences are likely. Since over 200 polymorphisms have been identified within CFTR itself [24], it is likely that some will interfere with its function, evidence for which is accumulating [25]. If factors such as these influence disease outcome then they were either under-represented or not influential enough in the older patient group, although they may provide some explanation for the patients who had evidence of preserved chloride secretion.

The other plausible explanation for the results from the present study relates to the characteristics of the groups studied. To investigate the relative differences in transepithelial ion transport a comparison was made with a significantly younger group with extremes of lung function (i.e. a surrogate marker of severity itself). Ion transport abnormalities are probably responsible for the abnormal airway environment which triggers the cycle of infection and inflammation, but whether or not it continues to drive this response is less clear. CFTR function was measured in the old group at an age that may have been many years after the initiation of this cycle and therefore may be less relevant to their survival. We also have made the assumption that the young patients with better lung function will themselves be long-term survivors, which of course can only be proven in 20 yrs time. Our study showed no correlation between age and measurements of ion transport but others have suggested that baseline PD decreases and sweat electrolytes increase as a function of age in CF $[4,11]$; further longitudinal studies are therefore required to evaluate this relationship.

The study was designed to assess whether patients with severe mutations have a survival advantage due to residual CFTR function and, therefore, by inclusion criteria, only patients with class I to III CFTR mutations were included. Thus it may be argued that, by definition, a significant chloride secretory response was not going to be demonstrated. However, the rationale for restricting the study to this genotype was threefold: 1) evidence of sporadic function exists in the literature for patients with such genotypes [9-11], and our results also confirmed this (table 3); 2) studies have shown that homozygous $\Delta$ F508 genotype can be associated with long-term survival and this may be due to variation in CFTR function, not environmental influences [12, 13]; and 3) increasingly the importance of $\mathrm{ENaC}$ and sodium transport on CF-like lung disease is being recognised [26]. This may also be directly relevant to $\mathrm{CF}$, as differences in the level of interaction between $\mathrm{ENaC}$ and CFTR may explain differences in the expression of 

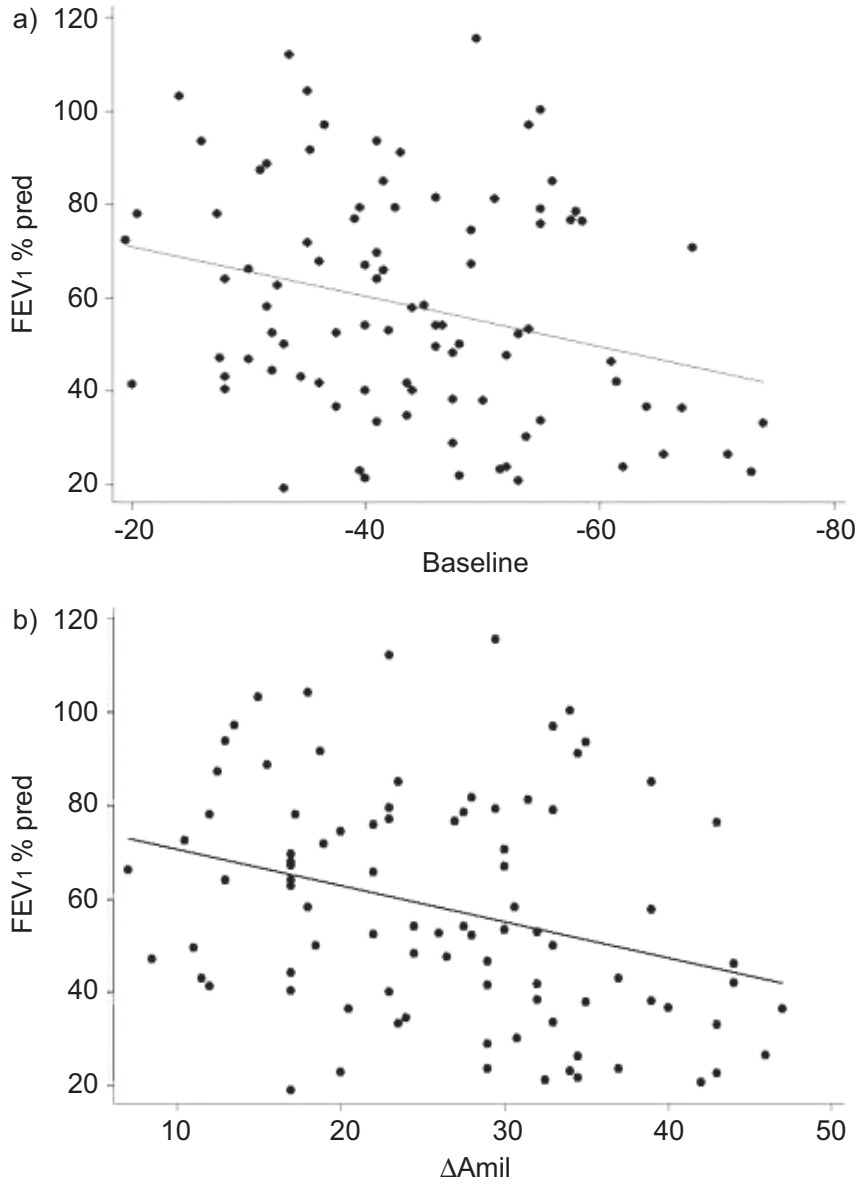

FIGURE 2. Association of a) baseline potential difference (PD) and b) change in $\mathrm{PD}$ in the presence of amiloride ( $\triangle \mathrm{Amil}$ ) with forced expiratory volume in $1 \mathrm{~s}$ (FEV 1$)$ on univariate analysis with coefficients $(95 \% \mathrm{Cl})-0.13(-0.23--0.03)$; $p=0.009$ and $-0.12(-0.20--0.04) ; p=0.003$, respectively. $\%$ pred: $\%$ predicted.

the disease and variability of survival. The study was powered to detect differences in baseline PD and therefore by restricting genotype we hoped to find evidence of these differences in $\mathrm{CF}$.
So what do these findings add to what is already known? First, they demonstrate that patients surviving for more than $40 \mathrm{yrs}$ with CF do not have preserved CFTR function at that timepoint compared with young patients. An association of lung function with $\Delta$ Amil was confirmed [11], but not with baseline PD, which has been demonstrated in previous studies [4, 14], although not consistently [27]. The chloride secretory response did not correlate with FEV1 or FVC, which is in contrast to findings from a study of $\Delta F 508$ homozygous sibling pairs [28] and a study showing residual function in males [10]. A recent study has demonstrated that the phenotype of airway transepithelial ion transport is a continuum and strongly associated with CFTR genotype [29]. Our study is consistent with this as we did not detect significant variations in CFTR function, presumably due to lack of genotype heterogeneity, but in line with their findings, sodium transport appears to be an important predictor of clinical phenotype, lending support to the $\mathrm{ENaC}$ hypothesis [26]. Our study also lends support to the presence of other important survival factors, independent of the initiating insult and ion channel abnormalities. One such factor is BMI; a striking finding was that BMI increased significantly in the old group between the ages of 20 and 40 yrs in spite of a significant decline in lung function, more $P$. aeruginosa and more CFRD. Interestingly, BMI of the longterm survivors at $20 \mathrm{yrs}$ of age was similar to the young severe group, despite lung function that was intermediate (i.e. between the young severe and mild groups) at that time. This suggests that survival for the mild group may exceed the older group which is in keeping with the steady increase in survival for each cohort of patients over the past few decades [30]. This is encouraging, but also serves to highlight the need for effective healthcare planning for the future as adult $\mathrm{CF}$ clinics continue to expand.

The sweat chloride results from the present study were unexpected, revealing a lower chloride concentration in the young/severe patients compared with the young/mild and older patients. Previous attempts to correlate sweat electrolytes with lung disease severity have been unsuccessful, citing random variation or environmental factors as possible explanations [16], which may also have been important in our study.

\begin{tabular}{|c|c|c|c|c|c|}
\hline & Baseline PD & $\Delta$ Amil & $\Delta \mathrm{LowCl}^{-}+$Iso & Wilschanski index & Sweat $\mathrm{Cl}^{-}$ \\
\hline FEV1 coefficient $(95 \% \mathrm{Cl})$ & $-0.21(-0.34--0.08)$ & $-0.14(-0.24--0.04)$ & $-0.03(-0.09-0.02)$ & $-0.001(-0.003-0.001)$ & $0.04(-0.05-0.13)$ \\
\hline $\mathrm{p}$-value & 0.002 & 0.007 & 0.20 & 0.33 & 0.38 \\
\hline Age coefficient $(95 \% \mathrm{Cl})$ & $-0.23(-0.47-0.01)$ & $-0.07(-0.26-0.13)$ & $-0.04(-0.14-0.06)$ & $-0.002(0.006-0.002)$ & $0.06(-0.11-0.23)$ \\
\hline $\mathrm{p}$-value & 0.25 & 0.49 & 0.38 & 0.90 & 0.44 \\
\hline BMI coefficient $(95 \% \mathrm{Cl})$ & $0.48(-0.38-1.34)$ & $0.11(-0.57-0.80)$ & $0.34(-0.02-0.71)$ & $0.01(-0.001-0.03)$ & $0.72(0.12-1.32)$ \\
\hline$p$-value & 0.27 & 0.75 & 0.06 & 0.07 & 0.02 \\
\hline
\end{tabular}




\begin{tabular}{|c|c|c|c|}
\hline \multirow{2}{*}{$\begin{array}{l}\text { TABLE } 3 \\
\text { Patient }\end{array}$} & \multicolumn{3}{|c|}{$\begin{array}{l}\text { Clinical characteristics of the three patients with } \\
\text { significant chloride secretion }\end{array}$} \\
\hline & 1 & 2 & 3 \\
\hline Age yrs (group) & $\begin{array}{l}23.3 \text { (young/ } \\
\text { severe) }\end{array}$ & $\begin{array}{l}21.5 \text { (young/ } \\
\text { severe) }\end{array}$ & 50.6 (old) \\
\hline Baseline PD mV & -32 & -44 & -43 \\
\hline$\Delta$ Amil mV & 22 & 39 & 37 \\
\hline$\Delta \mathrm{lowCl}^{-}+$Iso mV & -19 & -11 & -19 \\
\hline Wilschanski index & $0.42^{\#}$ & 0.75 & $0.60^{\#}$ \\
\hline Sweat $\mathrm{Cl}^{-} \mathrm{mmol} \cdot \mathrm{L}^{-1}$ & 90 & 96 & 100 \\
\hline Genotype & $\Delta \mathrm{F} 508 / \Delta \mathrm{F} 508$ & $\Delta \mathrm{F} 508 / \Delta \mathrm{F} 508$ & $\Delta \mathrm{F} 508 / \Delta \mathrm{F} 508$ \\
\hline Pancreatic status & $\mathrm{PI}$ & $\mathrm{PI}$ & PI \\
\hline FEV 1 \%pred & 53 & 58 & 21 \\
\hline CFRD & No & No & Yes \\
\hline $\begin{array}{c}\text { Pseudomonas } \\
\text { colonised }\end{array}$ & Yes & Yes & Yes \\
\hline \multicolumn{4}{|c|}{$\begin{array}{l}\text { Baseline PD: maximum stable potential difference; } \triangle \mathrm{Amil} \text { : change in PD in the } \\
\text { presence of amiloride; } \Delta \mathrm{lowCl}^{-}+\text {Iso: change in PD in the presence of low } \\
\text { chloride solution and isoprenaline; FEV1: forced expiratory volume in } 1 \mathrm{~s} \text {; } \\
\text { CFRD: cystic fibrosis related diabetes; PI: pancreatic insufficient. Wilschanski } \\
\text { index }=\mathrm{e}^{\text {response to low chloride solution and isoprenaline/response amiloride }} \text { [20]. }{ }^{\#} \text { : patient } \\
1 \text { and } 3 \text { details checked, as index }<0.7 \text {, and genotype verified as } \Delta \mathrm{F} 508 \text { / } \\
\Delta \mathrm{F} 508 \text {. }\end{array}$} \\
\hline
\end{tabular}

As the nasal PD values did not correlate with sweat chloride values in our study, it suggests that an undetermined mechanism may have interacted with the sweat physiology or, perhaps more probable in our opinion, that the sweat result was spurious and a result of a type I statistical error. Further limitations of our study might include the unequal sex distribution, as there is some evidence to suggest that nasal PD varies with menstruation [31]. In addition, the prevalence of

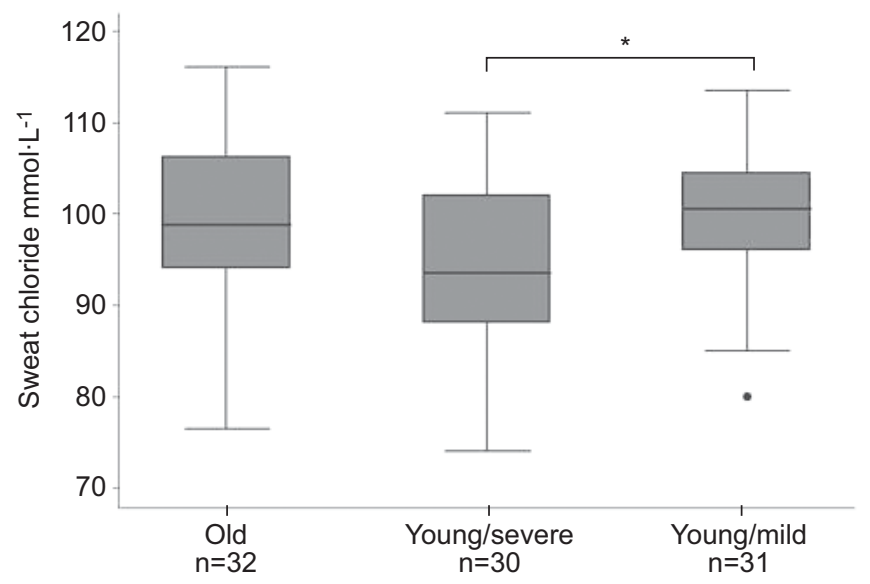

FIGURE 3. Box plot demonstrating the range and variability of sweat chloride concentrations for the three groups ( $p<0.05$; Kruskal-Wallis); the young/mild group had values which were significantly higher $(*: p<0.05)$ than the young/severe group. Boxes show the median and interquartile range (IQR). Whiskers show the highest and lowest data points within 1.5 IQR of the upper and lower quartile. - : outliers outside of these limits.

\section{TABLE 4 Nasal potential difference (PD) and sweat chloride reproducibility}

\begin{tabular}{|c|c|c|c|}
\hline Measurement & Mean difference & SD of difference & $\begin{array}{c}95 \% \text { limits of } \\
\text { agreement }\end{array}$ \\
\hline Baseline & 2.79 & 12.81 & $-22.33-27.90$ \\
\hline$\Delta$ Amil & 3.47 & 13.15 & $-29.24-22.31$ \\
\hline$\Delta$ low $\mathrm{Cl}^{-}$ & 1.12 & 6.83 & $-12.26-14.50$ \\
\hline$\Delta l o w C l^{-}+$Iso & 1.34 & 7.36 & $-13.08-15.76$ \\
\hline Sweat $\mathrm{Cl}^{-}$ & 2.78 & 5.04 & $-12.66-7.11$ \\
\hline
\end{tabular}

$\Delta$ Amil: change in $\mathrm{PD}$ in the presence of amiloride; $\Delta \mathrm{lowCl}^{-}$: change in $\mathrm{PD}$ in the presence of low chloride solution; $\Delta \mathrm{lowCl}^{-}+$Iso: change in $\mathrm{PD}$ in the presence of low chloride solution and isoprenaline.

$P$. aeruginosa infection was highest in the young severe group, the multivariate analysis from our study showed this variable was not associated with measures of ion transport, although others have demonstrated that this association may exist [15]. The intra-subject variability of nasal PD and sweat chloride measurements warrants acknowledgement. The mean difference and limits of agreement (table 4) show variability was present, particularly for measures of chloride secretion, and although this would have resulted in some loss of power, the variability was similar to other published studies [32].

This is the largest study of nasal PD measurements in "old" CF patients to date. By using an age criteria of $\geqslant 40 \mathrm{yrs}$ and specific genotypes, we identified a unique subset of CF patients who had lived beyond the current median life expectancy of approximately $38 \mathrm{yrs}$, which is not explained by "mild" mutations. Our findings indicate that long-term survival cannot be explained by residual CFTR function when measurements (nasal PD and sweat chloride) are taken in later life. This has important implications for other factors, such as BMI, which we postulate was one of the most significant influences of survival in this group [13]. Without onerous longitudinal studies to determine if patients with residual CFTR function and better lung function exceed life expectancy, we suggest that predictions of survival based on nasal PD and sweat test measurements should not be made.

\section{STATEMENT OF INTEREST}

None declared.

\section{REFERENCES}

1 Knowles MR, Stutts MJ, Spock A, et al. Abnormal ion permeation through cystic fibrosis respiratory epithelium. Science 1983; 221: 1067-1070.

2 Anderson MP, Gregory RJ, Thompson S, et al. Demonstration that CFTR is a chloride channel by alteration of its anion selectivity. Science 1991; 253: 202-205.

3 Stutts MJ, Canessa CM, Olsen JC, et al. CFTR as a cAMPdependent regulator of sodium channels. Science 1995; 269: 847-850.

4 Alton EW, Currie D, Logan-Sinclair R, et al. Nasal potential difference: a clinical diagnostic test for cystic fibrosis. Eur Respir J 1990; 3: 922-926. 
5 Knowles MR, Paradiso AM, Boucher RC. In vivo nasal potential difference: techniques and protocols for assessing efficacy of gene transfer in cystic fibrosis. Hum Gene Ther 1995; 6: 445-455.

6 Picard E, Aviram M, Yahav Y, et al. Familial concordance of phenotype and microbial variation among siblings with CF. Pediatr Pulmonol 2004; 38: 292-297.

7 Correlation between genotype and phenotype in patients with cystic fibrosis. The Cystic Fibrosis Genotype-Phenotype Consortium. N Engl J Med 1993; 329: 1308-1313.

8 Schechter MS. Non-genetic influences on cystic fibrosis lung disease: the role of sociodemographic characteristics, environmental exposures, and healthcare interventions. Semin Respir Crit Care Med 2003; 24: 639-652.

9 Ho LP, Samways JM, Porteous DJ, et al. Correlation between nasal potential difference measurements, genotype and clinical condition in patients with cystic fibrosis. Eur Respir J 1997; 10: 2018-2022.

10 Thomas SR, Jaffe A, Geddes DM, et al. Pulmonary disease severity in men with $\Delta \mathrm{F} 508$ cystic fibrosis and residual chloride secretion. Lancet 1999; 353: 984-985.

11 Leal T, Fajac I, Wallace HL, et al. Airway ion transport impacts on disease presentation and severity in cystic fibrosis. Clin Biochem 2008; 41: 764-772.

12 Simmonds NJ, Cullinan P, Hodson ME. Growing old with cystic fibrosis - the characteristics of long-term survivors of cystic fibrosis. Respir Med 2009; 103: 629-635.

13 Simmonds NJ, MacNeill SJ, Cullinan P, et al. Cystic fibrosis and survival to 40 years: a case-control study. Eur Respir J 2010; 36: 1277-1283.

14 Fajac I, Hubert D, Bienvenu T, et al. Relationships between nasal potential difference and respiratory function in adults with cystic fibrosis. Eur Respir J 1998; 12: 1295-1300.

15 Fajac I, Hubert D, Guillemot D, et al. Nasal airway ion transport is linked to the cystic fibrosis phenotype in adult patients. Thorax 2004; 59: 971-976.

16 Davis PB, Schluchter MD, Konstan MW. Relation of sweat chloride concentration to severity of lung disease in cystic fibrosis. Pediatr Pulmonol 2004; 38: 204-209.

17 De Boeck K, Wilschanski M, Castellani C, et al. Cystic fibrosis: terminology and diagnostic algorithms. Thorax 2006; 61: 627-635.

18 Gibson GJ. Standardised lung function testing. Eur Respir J 1993; 6: 155-157.
19 Middleton PG, Geddes DM, Alton EW. Protocols for in vivo measurement of the ion transport defects in cystic fibrosis nasal epithelium. Eur Respir J 1994; 7: 2050-2056.

20 Wilschanski M, Famini H, Strauss-Liviatan N, et al. Nasal potential difference measurements in patients with atypical cystic fibrosis. Eur Respir J 2001; 17: 1208-1215.

21 Mastella G, Di Cesare G, Borruso A, et al. Reliability of sweattesting by the Macroduct collection method combined with conductivity analysis in comparison with the classic Gibson and Cooke technique. Acta Paediatr 2000; 89: 933-937.

22 Wilschanski M, Dupuis A, Ellis L, et al. Mutations in the cystic fibrosis transmembrane regulator gene and in vivo transepithelial potentials. Am J Respir Crit Care Med 2006; 174: 787-794.

23 Mekus F, Ballmann M, Bronsveld I, et al. Categories of deltaF508 homozygous cystic fibrosis twin and sibling pairs with distinct phenotypic characteristics. Twin Res 2000; 3: 277-293.

24 Davies JC. Modifier genes in cystic fibrosis. Pediatr Pulmonol Suppl 2004; 26: 86-87.

25 Noone PG, Pue CA, Zhou Z, et al. Lung disease associated with the IVS8 5T allele of the CFTR gene. Am J Respir Crit Care Med 2000; 162: 1919-1924

26 Sheridan MB, Fong P, Groman JD, et al. Mutations in the $\beta$-subunit of the epithelial $\mathrm{Na}^{+}$channel in patients with a cystic fibrosis-like syndrome. Hum Mol Genet 2005; 14: 3493-3498.

27 Wallace HL, Barker PM, Southern KW. Nasal airway ion transport and lung function in young people with cystic fibrosis. Am J Respir Crit Care Med 2003; 168: 594-600.

28 Bronsveld I, Mekus F, Bijman J, et al. Chloride conductance and genetic background modulate the cystic fibrosis phenotype of Delta F508 homozygous twins and siblings. J Clin Invest 2001; 108: 1705-1715.

29 Bienvenu T, Sermet-Gaudelus I, Burgel P-R, et al. Cystic fibrosis transmembrane conductance regulator channel dysfunction in non-cystic fibrosis bronchiectasis. Am J Respir Crit Care Med 2010; 181: 1078-1084.

30 Dodge JA, Lewis PA, Stanton M, et al. Cystic fibrosis mortality and survival in the UK: 1947-2003. Eur Respir J 2007; 29: 522-526.

31 Sweezey NB, Smith D, Corey M, et al. Amiloride-insensitive nasal potential difference varies with the menstrual cycle in cystic fibrosis. Pediatr Pulmonol 2007; 42: 519-524.

32 Yaakov Y, Kerem E, Yahav Y, et al. Reproducibility of nasal potential difference measurements in cystic fibrosis. Chest 2007; 132: $1219-1226$ 